\title{
Peptidylarginine Deiminase Inhibitor Suppresses Neutrophil Extracellular Trap Formation and MPO-ANCA Production
}

\begin{abstract}
Yoshihiro Kusunoki', Daigo Nakazawa', Haruki Shida ${ }^{1}$, Fumihiko Hattanda ${ }^{1}$, Arina Miyoshi', Sakiko Masuda ${ }^{2}$, Saori Nishio', Utano Tomaru ${ }^{3}$, Tatsuya Atsumi' and Akihiro Ishizu ${ }^{2 *}$

'Division of Rheumatology, Endocrinology and Nephrology, Hokkaido University Graduate School of Medicine, Sapporo, Japan, ${ }^{2}$ Faculty of Health Sciences, Hokkaido University, Sapporo, Japan, ${ }^{3}$ Department of Pathology, Hokkaido University Graduate School of Medicine, Sapporo, Japan
\end{abstract}

Myeloperoxidase-antineutrophil cytoplasmic antibody (MPO-ANCA)-associated vasculitis is a systemic small-vessel vasculitis, wherein, MPO-ANCA plays a critical role in the pathogenesis. Neutrophil extracellular traps (NETs) released from activated neutrophils are composed of extracellular web-like DNA and antimicrobial proteins, including MPO.

OPEN ACCESS

Edited by: Marko Radic,

University of Tennessee, USA

Reviewed by:

Paul Proost,

University of Leuven, Belgium

Angelo A. Manfredi,

Vita Salute San Raffaele

University, Italy

Huw Lewis,

GlaxoSmithKline, UK

*Correspondence:

Akihiro Ishizu

aishizu@med.hokudai.ac.jp

Specialty section: This article was submitted to

Molecular Innate Immunity, a section of the journal

Frontiers in Immunology

Received: 17 March 2016 Accepted: 26 May 2016

Published: 08 June 2016

Citation:

Kusunoki Y, Nakazawa D, Shida H, Hattanda F, Miyoshi A, Masuda S, Nishio S, Tomaru U, Atsumi T and Ishizu A (2016) Peptidylarginine Deiminase Inhibitor Suppresses Neutrophil Extracellular Trap Formation and MPO-ANCA Production.

Front. Immunol. 7:227. doi: 10.3389/fimmu.2016.00227
Diverse stimuli, such as phorbol myristate acetate (PMA) and ligands of toll-like receptors (TLR), induce NETs. Although TLR-mediated NET formation can occur with preservation of living neutrophilic functions (called vital NETosis), PMA-stimulated neutrophils undergo cell death with NET formation (called suicidal NETosis). In the process of suicidal NETosis, histones are citrullinated by peptidylarginine deiminase 4 (PAD4). Since this step is necessary for decondensation of DNA, PAD4 plays a pivotal role in suicidal NETosis. Although NETs are essential for elimination of microorganisms, excessive formation of NETs has been suggested to be implicated in MPO-ANCA production. This study aimed to determine if pan-PAD inhibitors could suppress MPO-ANCA production in vivo. At first, NETs were induced in peripheral blood neutrophils derived from healthy donors $\left(1 \times 10^{6} / \mathrm{ml}\right)$ by stimulation with $20 \mathrm{nM}$ PMA with or without $20 \mu \mathrm{M}$ propylthiouracil (PTU), an anti-thyroid drug. We then determined that the in vitro NET formation was inhibited completely by $200 \mu \mathrm{M} \mathrm{Cl-amidine,} \mathrm{a} \mathrm{pan-PAD} \mathrm{inhibitor.} \mathrm{Next,} \mathrm{we} \mathrm{established}$ mouse models with MPO-ANCA production. BALB/c mice were given intraperitoneal (i.p.) injection of PMA (50 ng at days 0 and 7 ) and oral PTU ( $2.5 \mathrm{mg} /$ day) for 2 weeks. These mice were divided into two groups; the first group was given daily i.p. injection of PBS (200 $\mu \mathrm{l} /$ day) $(n=13)$ and the other group with daily i.p. injection of $\mathrm{Cl}$-amidine (0.3 mg/200 $\mu \mathrm{lPBS} /$ day) $(n=7)$. Two weeks later, citrullination as an indicator of NET formation in the peritoneum and serum MPO-ANCA titer was compared between the two groups. Results demonstrated that citrullination in the peritoneum was significantly reduced in the $\mathrm{Cl}$-amidine-treated mice compared with the vehicle-injected control mice (38\% reduction). Additionally, the serum MPO-ANCA titer of the Cl-amidine-treated mice $(32.3 \pm 31.0 \mathrm{ng} / \mathrm{ml})$ was significantly lower than that in the vehicle-injected mice $(132.1 \pm 41.6 \mathrm{ng} / \mathrm{ml})$. The collective findings indicate that excessive formation of NETs may be implicated in MPO-ANCA production in vivo.

Keywords: MPO-ANCA-associated vasculitis, MPO-ANCA, neutrophil extracellular trap, peptidylarginine deiminase 4, peptidylarginine deiminase inhibitor 


\section{INTRODUCTION}

Antineutrophil cytoplasmic antibody (ANCA)-associated vasculitis is a systemic small-vessel vasculitis (1). The major target antigens of ANCA are myeloperoxidase (MPO) and proteinase 3 (PR3). Neutrophils primed by pro-inflammatory cytokines, such as TNF- $\alpha$, express MPO and PR3 on the cell surface. ANCA bind to the antigens and then activate neutrophils directly and/ or through binding to bystander Fcy receptors. Consequently, the activated neutrophils induce vascular endothelial cell injury resulting in the development of small-vessel vasculitis $(2,3)$. ANCA, therefore, play a critical role in the pathogenesis of ANCA-associated vasculitis. Although the mechanism of MPO-ANCA production was unknown for a long time, recent studies have suggested the involvement of neutrophil extracellular traps (NETs) in the mechanism (4-6).

Neutrophil extracellular traps are firstly reported in 2004 as extracellular web-like DNA studded with antimicrobial proteins, including MPO, which are released from phorbol myristate acetate (PMA)-stimulated neutrophils (7). The PMA-stimulated neutrophils undergo cell death with the formation of NETs (8), though not all stimuli induce cell death in NET-forming neutrophil (9). It has been demonstrated that NET formation can occur with preservation of living neutrophilic functions, including phagocytosis and chemotaxis $(10,11)$. Currently, NET formation undergoing cell death is called suicidal NETosis, whereas that preserves living neutrophilic functions is called vital NETosis. NETs can trap microorganisms by the extracellular DNA and kill them using the antimicrobial proteins. Thus, NETosis is considered as an important event in innate immunity. However, excessive NETosis can result in vascular endothelial cell injury (12), thrombosis (13, $14)$, and impairment of diabetic wound healing $(15,16)$. In addition, disordered regulation of NETosis has been suggested to be involved in the pathogenesis of autoimmune diseases, including systemic lupus erythematosus (SLE) (17) and anti-thyroid drug propylthiouracil (PTU)-induced MPO-ANCA-associated vasculitis (5). In the last two studies, the possibility of extracellular components in persistent NETs recognized as autoantigens by the immune system was discussed.

Suicidal NETosis is induced in response to diverse stimuli, including PMA $(7,18)$. These stimuli activate the Raf-mitogenactivated protein kinase kinase-extracellular signal-regulated kinase pathway, NADPH oxidase-dependent production of reactive oxygen species, and receptor-interacting protein kinase/ mixed lineage kinase domain-like-mediated signals $(19,20)$. In this pathway, peptidylarginine deiminase 4 (PAD4) yields citrullination of histones, around which DNA coils.

The PAD enzymes convert arginine residues to citrulline in a variety of protein substrates (21). Among the PAD family, which includes PAD 1-4 and 6, PAD4 is expressed mainly in hematopoietic cells, such as neutrophils (22). In the process of suicidal NETosis, PAD4-dependent citrullination of histones that yields decondensation of DNA is an essential step to mix DNA and intracytoplasmic proteins. This mixture is subsequently extruded from the ruptured plasma membrane. Accordingly, PAD4 plays a pivotal role in the process of suicidal NETosis (23).
In the present study, we aimed to determine if a pan-PAD inhibitor, $\mathrm{Cl}$-amidine, could suppress MPO-ANCA production in vivo. For this purpose, we attempted to generate novel mouse models of MPO-ANCA-associated vasculitis according to our previous protocol utilized for establishment of a rat model of this disease (5). The mouse models would be more useful models that require lower doses of reagents than the rat model.

\section{MATERIALS AND METHODS}

\section{Human Neutrophil Isolation}

Human peripheral blood neutrophils were obtained from healthy volunteers by density centrifugation using Polymorphprep (Axis-Shield, Dundee, Scotland) according to the manufacturer's instructions.

\section{NET Induction In Vitro}

The in vitro NET induction was conducted similarly to our earlier study (5). In brief, human peripheral blood neutrophils were re-suspended in RPMI 1640 medium supplemented with $5 \%$ fetal bovine serum and then seeded in wells of 4 -well chamber slides (Thermo Fisher Scientific, Yokohama, Japan) $\left(1 \times 10^{6} / \mathrm{ml}\right)$. After incubation for $30 \mathrm{~min}$ at $37^{\circ} \mathrm{C}$, the cells were exposed to 0 or 20 nM PMA (Sigma-Aldrich, St. Louis, MO, USA) with or without $20 \mu \mathrm{M}$ PTU (Chugai Pharmaceutical, Tokyo, Japan) and incubated for another $2 \mathrm{~h}$ at $37^{\circ} \mathrm{C}$.

\section{PAD Inhibitor Administration In Vitro}

Fifteen minutes prior to the PMA/PTU administration, $200 \mu \mathrm{M}$ Cl-amidine (Calbiochem, San Diego, CA, USA), a pan-PAD inhibitor, was added alternately into the wells. The concentration of $\mathrm{Cl}$-amidine was adopted according to the previous report (24).

\section{Quantification of NETs In Vitro}

After $2 \mathrm{~h}$ of incubation, the medium containing the reagents was removed, and the remaining cells were washed with PBS followed by fixation with $4 \%$ paraformaldehyde for $15 \mathrm{~min}$. Thereafter, the specimens were made to react with $5 \mu \mathrm{g} / \mathrm{ml}$ of rabbit anti-human citrullinated histone 3 polyclonal antibody (Abcam, Cambridge, UK) for $60 \mathrm{~min}$ at room temperature. After removal of unbound antibody, the specimens were next allowed to react with 1:500 dilution of Alexa Fluor 594-conjugated goat anti-rabbit IgG antibody (Invitrogen, Tokyo, Japan) for $60 \mathrm{~min}$ at room temperature. After washing with PBS, the specimens were finally mounted with the 4', 6-diamidino-2-phenylindole (DAPI)-containing solution (Sigma-Aldrich). NET formation was observed under a fluorescent microscope and was quantified by counting the citrullinated histone 3-positive cells per $\times 100$ power field of view. Data from five random fields of view $(\times 100)$ were subjected to the quantitative analysis.

\section{Establishment of Mouse Models with MPO-ANCA Production}

BALB/c, New Zealand White (NZW), C57BL/6N (B6/N), C57BL/6J (B6/J), and DBA mice (14-week-old female) were purchased from Clea Japan (Tokyo, Japan). The mice were given 
intraperitoneal (i.p.) injection of PMA (50 ng at days 0 and 7) and oral administration of PTU dissolved in $5 \%$ glucose water for 4 weeks ( $n=5 /$ strain). Since preliminary experiments revealed that each mouse ingested at least $2.5 \mathrm{ml}$ of the drug-containing glucose water, the concentration of PTU was set as $1 \mathrm{mg} / \mathrm{ml}$ in order to administer exactly $2.5 \mathrm{mg}$ PTU per day. These mice were maintained under specific pathogen-free condition in accordance with the guidelines for the care and use of laboratory animals in Hokkaido University (Permission No. 12-0077).

\section{Laboratory Data}

Mouse urine was collected during the last $24 \mathrm{~h}$ using metabolic cages. Blood samples were obtained at days 14 and 28. Hematuria was assessed by dipsticks (Siemens Healthcare, Tokyo, Japan). Biochemical examinations for blood nitrogen urea (BUN) and creatinine $(\mathrm{Cr})$ were performed at Daiichi Kishimoto Clinical Laboratory (Sapporo, Japan). Serum titer of MPO-ANCA was determined by enzyme-linked immunosorbent assay (ELISA) at A-CLIP Institute (Chiba, Japan).

\section{Histological Evaluation}

The lungs, kidneys, and peritoneal tissues were obtained at day 28 and then fixed in $10 \%$ formalin. The pulmonary and renal sections were subjected to hematoxylin and eosin staining. The sections of the peritoneal tissues were subjected to immunohistochemistry for citrullinated histone 3 as described previously $(25,26)$.

\section{PAD Inhibitor Administration In Vivo}

$\mathrm{BALB} / \mathrm{c}$ mice (14-week-old female) were given i.p. injection of PMA (50 ng at days 0 and 7) and oral PTU (2.5 mg/day, aforementioned protocol) for 2 weeks. These mice were divided into two groups. The first group was given daily i.p. injection of PBS $(200 \mu \mathrm{l} /$ day $)(n=13)$. The second group was given daily i.p. injection of $\mathrm{Cl}$-amidine $(0.3 \mathrm{mg} / 200 \mu \mathrm{l} \mathrm{PBS} /$ day $)(n=7)$. The dose of $\mathrm{Cl}$-amidine was adopted according to the previous report (27). Each mouse was administered at least $10 \mathrm{mg} / \mathrm{kg}$ of $\mathrm{Cl}$-amidine per day. Mouse urine was collected during the last $24 \mathrm{~h}$ using metabolic cages. Blood and tissue samples were obtained at day 14 .

\section{Statistical Analysis}

The in vitro data were presented as mean \pm standard deviation (SD) values obtained from experiments repeated for at least five times. The in vivo data were also presented as mean \pm SD values. Mann-Whitney $U$-tests were applied for statistical evaluation with StatPlus software. $P$-values of $<0.05$ were regarded as statistically significant.

\section{RESULTS}

\section{Effect of PAD Inhibitor on NET Induction In Vitro}

Human peripheral blood neutrophils were exposed to 0 or $20 \mathrm{nM}$ PMA with or without $20 \mu \mathrm{M}$ PTU. Fifteen minutes prior to PMA/ PTU exposure, the neutrophils were treated with or without $200 \mu \mathrm{M} \mathrm{Cl}$-amidine. Stimulation without PMA did not induce
NETs (data not shown). It has been also determined that $200 \mu \mathrm{M}$ of Cl-amidine did not affect the viability of neutrophils in vitro (Figure S1 in Supplementary Material).

The neutrophils stimulated by $20 \mathrm{nM}$ PMA alone formed extended NETs, whereas neutrophils stimulated by $20 \mathrm{nM}$ PMA plus $20 \mu \mathrm{M}$ PTU formed non-extended round-shaped NETs (Figure 1A). These findings corresponded with our earlier observations (5) and suggested that PTU yielded abnormal conformation of NETs induced by PMA. Although the morphology of NETs was different, both stimuli (PMA alone and PMA plus PTU) were shown to induce citrullination of histone 3 (5). Therefore, PAD4 was likely involved in the NET induction by these stimuli. As expected, the pan-PAD inhibitor, Cl-amidine $(200 \mu \mathrm{M})$ inhibited the NET formation induced by both PMA alone and PMA plus PTU in vitro (Figures 1A,B). Furthermore,

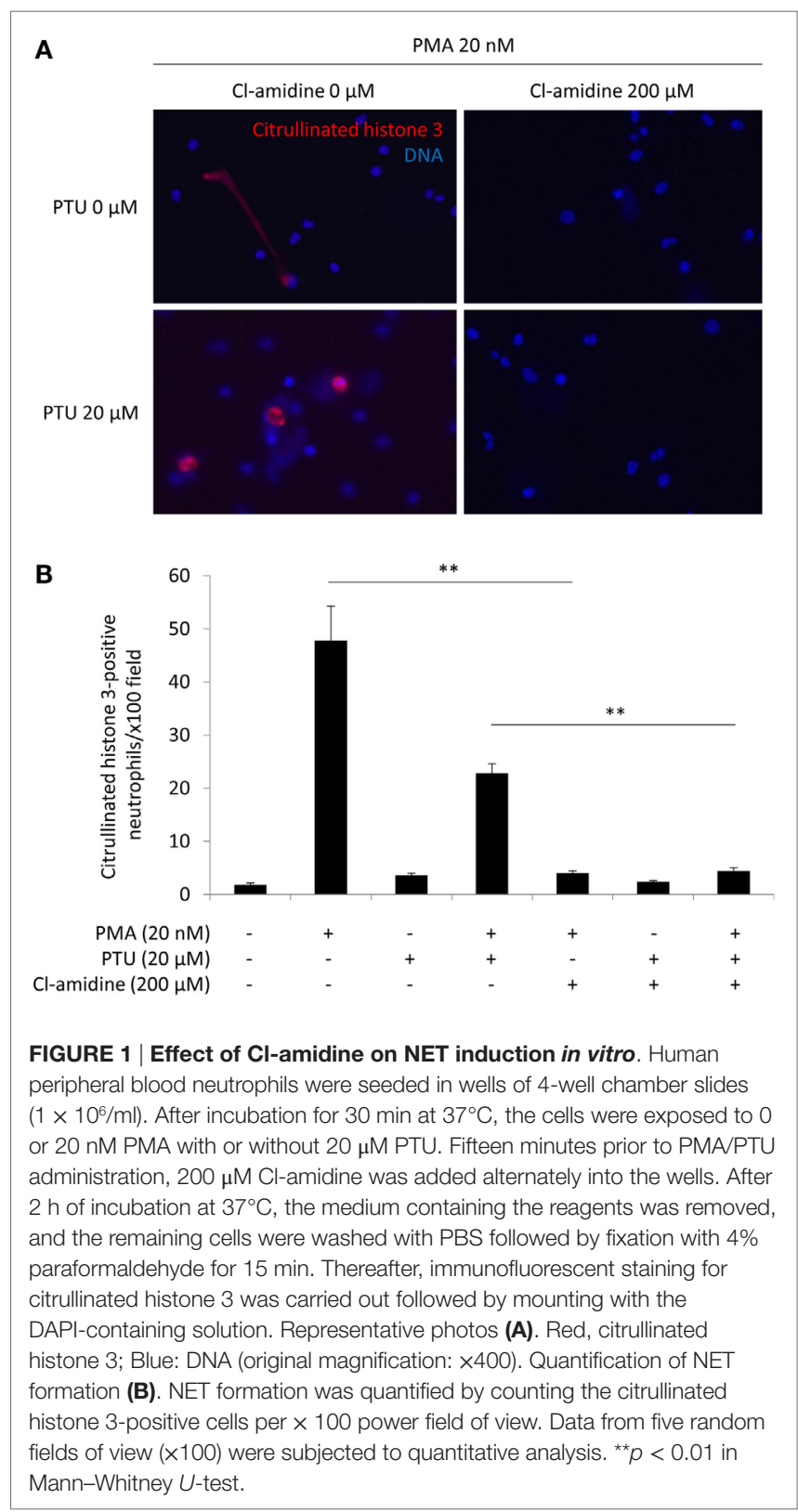


these findings suggest that $\mathrm{Cl}$-amidine can inhibit not only the formation of PMA-induced conventional (extended) NETs but also PTU-mediated unconventional (non-extended roundshaped) NETs.

\section{Establishment of Mouse Models with MPO-ANCA Production}

In our earlier study, WKY rats were employed to establish an animal model of MPO-ANCA-associated vasculitis (5). In this study, we attempted to establish novel mouse models of MPOANCA-associated vasculitis according to the protocol for the rat model. For this purpose, BALB/c, NZW, B6/N, B6/J, and DBA mice ( $n=5 /$ strain) were given i.p. injection of PMA (50 ng at days 0 and 7) and oral PTU (2.5 mg/day) for 4 weeks (Figure 2A). The serum titers of MPO-ANCA at day 28 were $100.4 \pm 12.0 \mathrm{ng} /$ $\mathrm{ml}$ in $\mathrm{BALB} / \mathrm{c}, 96.1 \pm 12.8 \mathrm{ng} / \mathrm{ml}$ in $\mathrm{NZW}, 41.3 \pm 0.90 \mathrm{ng} / \mathrm{ml}$ in $\mathrm{B} 6 / \mathrm{N}, 31.6 \pm 5.79 \mathrm{ng} / \mathrm{ml}$ in $\mathrm{B} 6 / \mathrm{J}$, and $32.0 \pm 4.06 \mathrm{ng} / \mathrm{ml}$ in

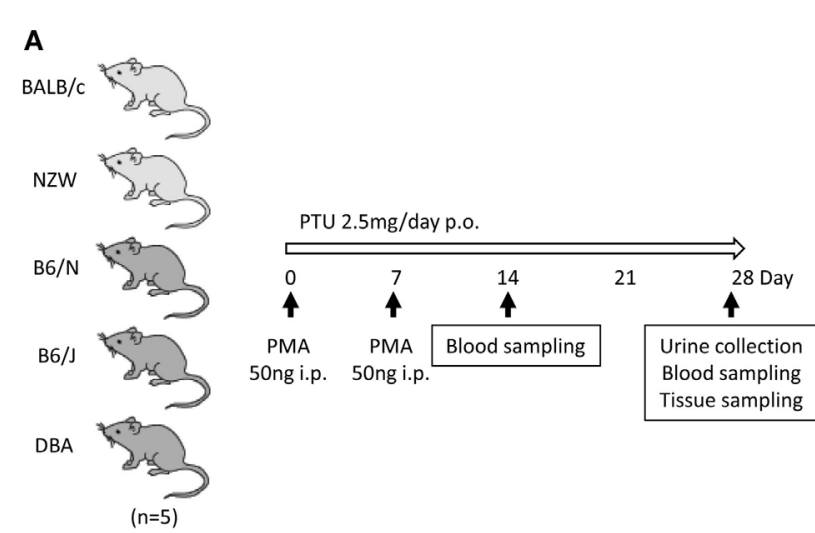

B

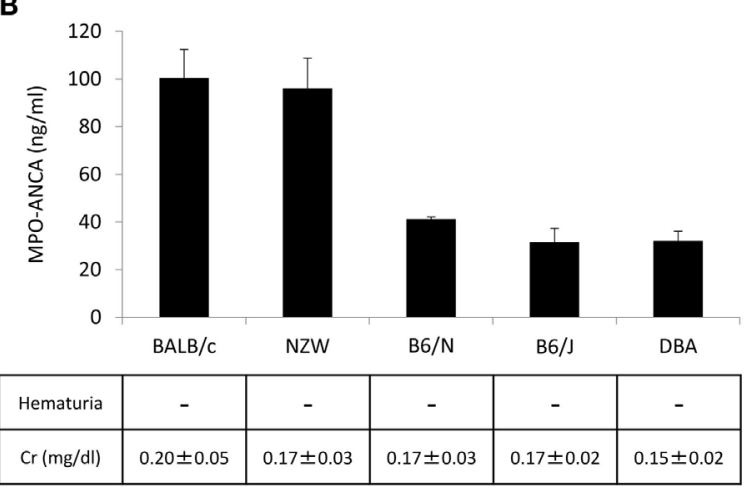

FIGURE 2 | Establishment of mouse models with MPO-ANCA production. Experimental protocol (A). BALB/c, NZW, B6/N, B6/J, and DBA mice (14-week-old female) were given i.p. injection of PMA (50 ng at days 0 and 7 ) and oral PTU (2.5 mg/day) for 4 weeks ( $n=5 /$ strain, p.o.: Per Os). Mouse urine was collected during the last $24 \mathrm{~h}$ using metabolic cages, and then hematuria was assessed by dipsticks. Blood samples were obtained at days 14 and 28, and then the concentrations of BUN and $\mathrm{Cr}$ were determined. The lungs, kidneys, and peritoneal tissues were obtained at day 28. Serum titers of MPO-ANCA determined by ELISA were shown with the results of the urinalysis and concentrations of $\mathrm{Cr}$ in the serum (B). The normal limit of serum Cr level is $0.4 \mathrm{mg} / \mathrm{dl}$.
DBA mice (Figure 2B). Contrary to the rat model, no vasculitic lesion was observed in the lungs and kidneys of all mouse strains examined. Correspondingly, renal dysfunction was not detected in the urine and blood samples. Based on these findings, we employed $\mathrm{BALB} / \mathrm{c}$ mice to construct the mouse model with MPO-ANCA production. The serum MPO-ANCA titer at day 14 was $79.0 \pm 5.70 \mathrm{ng} / \mathrm{ml}$ in the BALB/c model. MPO-ANCA was not detected in the vehicle-injected $B A L B / c$ mice.

\section{Effect of PAD Inhibitor on Citrullination In Vivo}

$\mathrm{BALB} / \mathrm{c}$ mice (14-week-old female) were given i.p. injection of PMA (50 ng at days 0 and 7) and oral PTU (2.5 mg/day) for 2 weeks. These mice were divided into two groups. The first group was given daily i.p. injection of PBS $(200 \mu \mathrm{l} /$ day $)(n=13)$ and the other group with daily i.p. injection of $\mathrm{Cl}$-amidine $(0.3 \mathrm{mg} / 200 \mu \mathrm{l}$ $\mathrm{PBS} /$ day) $(n=7)$ (Figure 3A). At day 14, the peritoneal tissues were subjected to immunohistochemistry for citrullinated histone 3 for the evaluation of NET formation in vivo. As a result, the rate of citrullinated histone 3-positive cells in polymorphonuclear cells was significantly reduced in the Cl-amidine-treated mice in comparison with the vehicle-injected control mice $(38 \%$ reduction) (Figure $3 \mathbf{B}$ ).

\section{Effect of PAD Inhibitor on MPO-ANCA Production In Vivo}

The serum titers of MPO-ANCA in the Cl-amidine-treated mice $(32.3 \pm 31.0 \mathrm{ng} / \mathrm{ml})$ were significantly lower than the vehicleinjected control mice $(132.1 \pm 41.6 \mathrm{ng} / \mathrm{ml})$ (Figure 3C). The collective findings clearly indicate that NET formation is inhibited by the pan-PAD inhibitor, $\mathrm{Cl}$-amidine, both in vitro and in vivo, and that MPO-ANCA production is suppressed by $\mathrm{Cl}$-amidine in vivo.

\section{DISCUSSION}

The PMA-stimulated neutrophils extrude decondensated DNA, which forms extracellular web-like structures decorated with bactericidal proteins (7). Since this substance, called NETs, can bind and kill bacteria, NET formation is regarded as an important event in innate immunity. Chronic granulomatous disease (CGD) patients who cannot generate NETs are susceptible to diverse bacteria and fungi indeed, and it was shown that restoration of NET formation in CGD resulted in resistance to such infections (28). Currently, it is considered that NET appears to be a form of innate response that binds microorganisms, prevents them from spreading, and ensures a high local concentration of antimicrobial agents derived from neutrophils (29).

The PMA-stimulated neutrophils undergo cell death with NET formation (8). Since the characteristics of cell death resembled neither typical necrosis nor apoptosis, Steinberg et al. coined NETosis for the neutrophil death with NET formation (30). However, Clark et al. have demonstrated that neutrophils do not necessarily undergo cell death after NET formation (31). It has been shown that NET formation can occur with preservation of neutrophilic functions, including phagocytosis and 
A

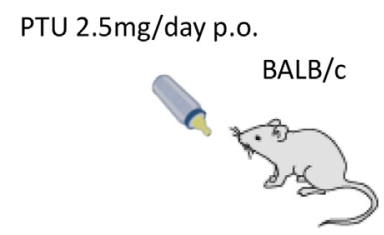

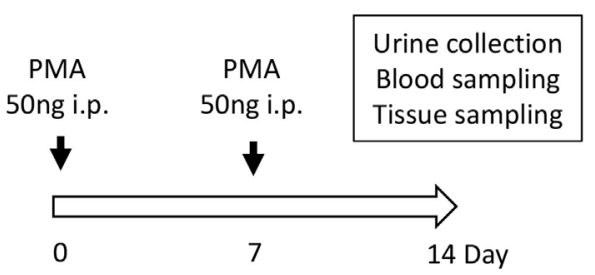

Daily i.p. of vehicle ( $n=13)$ or $\mathrm{Cl}$-amidine $(0.3 \mathrm{mg} / 200 \mu \mathrm{PBS}, \mathrm{n}=7)$

B
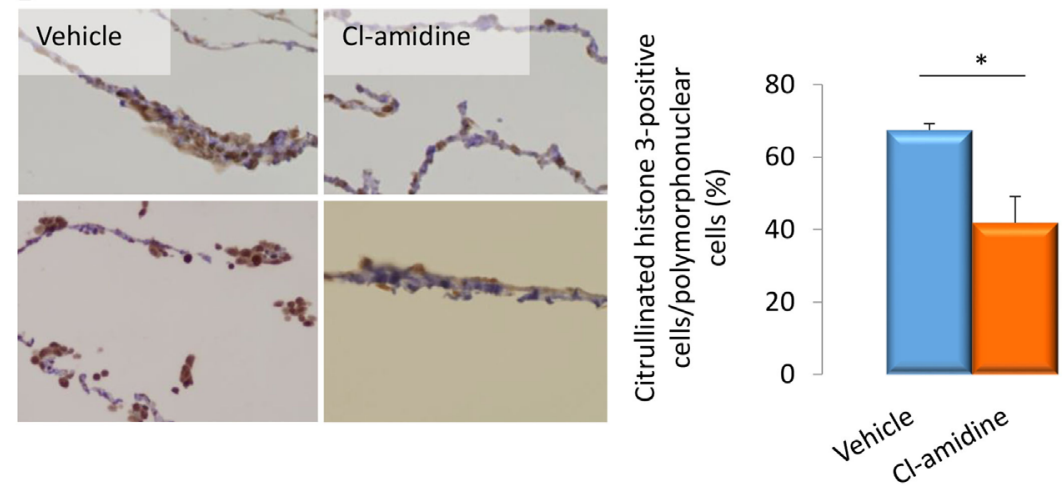

C

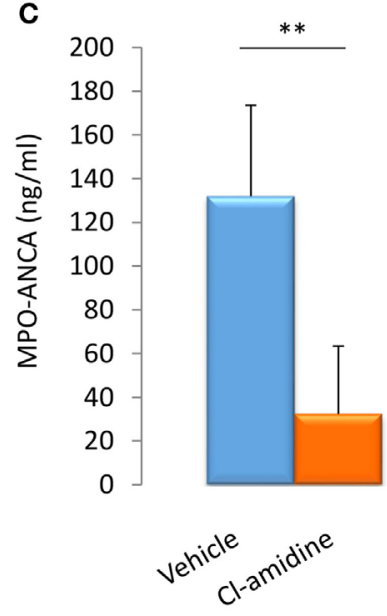

FIGURE 3 | Effect of Cl-amidine on citrullination and MPO-ANCA production in vivo. Experimental protocol (A). BALB/c mice (14-week-old female) were given i.p. injection of PMA (50 ng at days 0 and 7) and oral PTU (2.5 mg/day) for 2 weeks (p.o.: Per Os). These mice were divided into two groups. The first group of mice was given daily i.p. injection of PBS $(200 \mu \mathrm{l} /$ day) $(n=13)$. The other group was given daily i.p. injection of Cl-amidine $(0.3 \mathrm{mg} / 200 \mu \mathrm{l} \mathrm{PBS} /$ day) $(n=7)$. Mouse urine was collected during the last $24 \mathrm{~h}$ using metabolic cages. Blood and tissue samples were obtained at day 14 . NET formation in peritoneal tissues (B). The formalin-fixed paraffin-embedded sections of peritoneal tissues were subjected to immunohistochemistry for citrullinated histone 3 . Representative photos among five random fields of view $(\times 100)$ were shown. NET induction was quantified by calculating the rate of citrullinated histone 3-positive cells in polymorphonuclear cells in the five random fields of view. ${ }^{*} p<0.05$ in Mann-Whitney U-test. Serum titers of MPO-ANCA determined by ELISA (C). ${ }^{* *} p<0.01$ in Mann-Whitney U-test.

chemotaxis $(10,11)$. This phenomenon termed vital NETosis (9). On the contrary, aforementioned dying NETosis has been called suicidal NETosis.

Although NETs play an essential role in the innate immunity, some harmful aspects of NETs have been demonstrated $(32,33)$. They not only have direct cytotoxic and thrombotic effects on vascular endothelium (12-14), but NETs are also associated with pathogenic autoantibody production $(5,17)$. For example, impairment of NET degradation potential (low activity of DNase I) in the serum is present in $30-40 \%$ of patients with SLE and is suggested to lead the production of anti-DNA antibodies, which are related to disease severity (17). In another case, morphologically abnormal and DNase I-resistant NETs are generated by the anti-thyroid drug, PTU, and then MPO in the NETs seems to be recognized by the immune system resulting in the production of MPO-ANCA in rats administered with PTU (5). It has been shown that a part of PTU is metabolized by MPO. Simultaneously, conformational alteration of MPO could be induced by PTU (34), which could result in the tolerance break to MPO. 
In the present study, we established mouse models with MPO-ANCA production. We treated five strains of mouse with PMA and PTU corresponding to the previously established protocol for the rat model of MPO-ANCA-associated vasculitis. Interestingly, there was a strain-dependency in the response to produce MPO-ANCA in mouse. Although the precise mechanism of the strain-dependency has to be determined in future studies, the Th2 phenotype of BALB/c (35) and autoimmuneprone genetic background of NZW (36) may be associated with the susceptibility to produce autoantibodies. In addition, no vasculitic lesion was observed in the mouse models with MPO-ANCA production, whereas WKY rats with MPO-ANCA developed pulmonary capillaritis and glomerulonephritis (5). Species-dependency may be present in the development of PMA plus PTU-induced MPO-ANCA-associated vasculitis. Nonetheless, we employed $\mathrm{BALB} / \mathrm{c}$ mice to construct in vivo models of MPO-ANCA production.

Neeli et al. first demonstrated that PAD4-dependent histone deimination occurred in activated neutrophils under inflammatory conditions (37). Since PAD4-deficient neutrophils did not generate NETs in response to PMA, PAD4 plays a pivotal role in the NET formation (23). Correspondingly, inhibition of PAD4 using the pan-PAD inhibitor, $\mathrm{Cl}$-amidine, prevented citrullination of histone 3 and significantly reduced NET release from HL60 cells, which were differentiated into mature neutrophils, in response to $\mathrm{Ca}^{2+}$ ionophore or Shigella flexneri exposure (24). In addition, $\mathrm{Cl}$-amidine has been shown to suppress NET formation in lupusprone mice (27). It has been shown that $\mathrm{Cl}$-amidine can modify the cysteine of PAD and then inactivate it irreversibly (38). Based on these findings, we conducted in vitro and in vivo experiments to inhibit citrullination/NET formation using $\mathrm{Cl}$-amidine as a pan-PAD inhibitor. The present study reproduced and extended the results of previous reports that investigated $\mathrm{Cl}$-amidine both in vitro and in vivo and demonstrated that MPO-ANCA production was suppressed by $\mathrm{Cl}$-amidine in vivo. These findings suggest that excessive formation of NETs may be implicated in MPOANCA production in vivo. In order to demonstrate the direct implication of PAD4-dependent NET formation in MPO-ANCA production, further studies using PAD4-deficient mice should be designed.

One limitation of this study is the lack of quantification of released NETs, which can be usually detected as MPO-DNA complexes in the serum. However, it has been shown that the PMA plus PTU-induced NETs hardly converted into soluble form (5). Thus, there is no better methodology to evaluate NETs in the

\section{REFERENCES}

1. Jennette JC, Falk RJ, Bacon PA, Basu N, Cid MC, Ferrario F, et al. 2012 revised international chapel hill consensus conference nomenclature of vasculitides. Arthritis Rheum (2013) 65:1-11. doi:10.1002/art.37715

2. Jennette JC, Falk RJ. Pathogenesis of antineutrophil cytoplasmic autoantibody-mediated disease. Nat Rev Rheumatol (2014) 10:463-73. doi:10.1038/ nrrheum.2014.103

3. Kallenberg CG. Pathogenesis and treatment of ANCA-associated vasculitides. Clin Exp Rheumatol (2015) 33:S11-4.

4. Kessenbrock K, Krumbholz M, Schonermarck U, Back W, Gross WL, Werb Z, et al. Netting neutrophils in autoimmune small-vessel vasculitis. Nat Med (2009) 15:623-5. doi:10.1038/nm.1959 murine model than the immunohistochemistry for citrullinated histone 3.

No vasculitic phenotype was observed in the PMA plus PTU-induced mouse models with MPO-ANCA production. Therefore, we could not examine the effect of PAD inhibitors on MPO-ANCA-associated vasculitis in this model, which is another limitation of this study. It should be determined whether increased doses of PMA/PTU and/or longer duration could induce vasculitis in the mouse models in future studies.

It has been shown that MPO-ANCA is the major pathogenic factor in MPO-ANCA-associated vasculitis $(2,3)$. Thus, it is expected that PAD inhibitors, which can suppress the production of the pathogenic autoantibody, would be applied for the treatment of patients with MPO-ANCA-associated vasculitis. Preceding studies have demonstrated the protective effects of PAD inhibitors on the models of SLE (27) and other NET-related diseases, including multiple sclerosis (39), collagen-induced arthritis (40), and inflammatory bowel disease (41). Although further studies are needed to clarify their safety and effectiveness, PAD inhibitors are potential candidates as novel therapeutic agents for various NET-related diseases, including MPO-ANCAassociated vasculitis.

\section{AUTHOR CONTRIBUTIONS}

YK, HS, FH, and AM performed the experiments. YK, DN, SM, SN, UT, TA, and AI analyzed and discussed the data. YK, DN, UT, and AI designed the research. YK, UT, and AI wrote the manuscript.

\section{ACKNOWLEDGMENTS}

The authors would like to thank Ms. Makiko Miwa for the technical assistance. This study was supported by grants from the Ministry of Education, Culture, Sports, Science and Technology of Japan [26293082 (AI)], Ministry of Health, Labour and Welfare of Japan [Research on Rare and Intractable Vasculitis (AI)], Japan Agency for Medical Research and Development [15ek0109104 and 15ek0109121 (AI)], and Akiyama Life Science Foundation (AI).

\section{SUPPLEMENTARY MATERIAL}

The Supplementary Material for this article can be found online at http://journal.frontiersin.org/article/10.3389/fimmu.2016.00227

5. Nakazawa D, Tomaru U, Suzuki A, Masuda S, Hasegawa R, Kobayashi T, et al. Abnormal conformation and impaired degradation of propylthiouracilinduced neutrophil extracellular traps: implications of disordered neutrophil extracellular traps in a rat model of myeloperoxidase antineutrophil cytoplasmic antibody-associated vasculitis. Arthritis Rheum (2012) 64:3779-87. doi:10.1002/art.34619

6. Sangaletti S, Tripodo C, Chiodoni C, Guarnotta C, Cappetti B, Casalini P, et al. Neutrophil extracellular traps mediate transfer of cytoplasmic neutrophil antigens to myeloid dendritic cells toward ANCA induction and associated autoimmunity. Blood (2012) 120:3007-18. doi:10.1182/blood-2012-03-416156

7. Brinkmann V, Reichard U, Goosmann C, Fauler B, Uhlemann Y, Weiss DS, et al. Neutrophil extracellular traps kill bacteria. Science (2004) 303:1532-5. doi:10.1126/science.1092385 
8. Fuchs TA, Abed U, Goosmann C, Hurwitz R, Schulze I, Wahn V, et al. Novel cell death program leads to neutrophil extracellular traps. J Cell Biol (2007) 176:231-41. doi:10.1083/jcb.200606027

9. Yipp BG, Kubes P. NETosis: how vital is it? Blood (2013) 122:2784-94. doi:10.1182/blood-2013-04-457671

10. Pilsczek FH, Salina D, Poon KK, Fahey C, Yipp BG, Sibley CD, et al. A novel mechanism of rapid nuclear neutrophil extracellular trap formation in response to Staphylococcus aureus. J Immunol (2010) 185:7413-25. doi:10.4049/jimmunol.1000675

11. Yipp BG, Petri B, Salina D, Jenne CN, Scott BN, Zbytnuik LD, et al. Infectioninduced NETosis is a dynamic process involving neutrophil multitasking in vivo. Nat Med (2012) 18:1386-93. doi:10.1038/nm.2847

12. Xu J, Zhang X, Pelayo R, Monestier M, Ammollo CT, Semeraro F, et al. Extracellular histones are major mediators of death in sepsis. Nat Med (2009) 15:1318-21. doi: $10.1038 / \mathrm{nm} .2053$

13. Fuchs TA, Brill A, Duerschmied D, Schatzberg D, Monestier M, Myers DD Jr, et al. Extracellular DNA traps promote thrombosis. Proc Natl Acad Sci U S A (2010) 107:15880-5. doi:10.1073/pnas.1005743107

14. Fuchs TA, Brill A, Wagner DD. Neutrophil extracellular trap (NET) impact on deep vein thrombosis. Arterioscler Thromb Vasc Biol (2012) 32:1777-83. doi:10.1161/ATVBAHA.111.242859

15. Wong SL, Demers M, Martinod K, Gallant M, Wang Y, Goldfine AB, et al. Diabetes primes neutrophils to undergo NETosis, which impairs wound healing. Nat Med (2015) 21:815-9. doi:10.1038/nm.3887

16. Fadini GP, Menegazzo L, Rigato M, Scattolini V, Poncina N, Bruttocao A, et al. NETosis delays diabetic wound healing in mice and humans. Diabetes (2016) 65(4):1061-71. doi:10.2337/db15-0863

17. Hakkim A, Furnrohr BG, Amann K, Laube B, Abed UA, Brinkmann V, et al. Impairment of neutrophil extracellular trap degradation is associated with lupus nephritis. Proc Natl Acad Sci U S A (2010) 107:9813-8. doi:10.1073/ pnas.0909927107

18. Yan J, Meng X, Wancket LM, Lintner K, Nelin LD, Chen B, et al. Glutathione reductase facilitates host defense by sustaining phagocytic oxidative burst and promoting the development of neutrophil extracellular traps. J Immunol (2012) 188:2316-27. doi:10.4049/jimmunol.1102683

19. Hakkim A, Fuchs TA, Martinez NE, Hess S, Prinz H, Zychlinsky A, et al. Activation of the Raf-MEK-ERK pathway is required for neutrophil extracellular trap formation. Nat Chem Biol (2011) 7:75-7. doi:10.1038/ nchembio. 496

20. Desai J, Kumar SV, Mulay SR, Konrad L, Romoli S, Schauer C, et al. PMA and crystal-induced neutrophil extracellular trap formation involves RIPK1RIPK3-MLKL signaling. Eur J Immunol (2016) 46:223-9. doi:10.1002/ eji.201545605

21. Rohrbach AS, Slade DJ, Thompson PR, Mowen KA. Activation of PAD4 in NET formation. Front Immunol (2012) 3:360. doi:10.3389/fimmu.2012.00360

22. Wang S, Wang Y. Peptidylarginine deiminases in citrullination, gene regulation, health and pathogenesis. Biochim Biophys Acta (2013) 1829:1126-35. doi:10.1016/j.bbagrm.2013.07.003

23. Li P, Li M, Lindberg MR, Kennett MJ, Xiong N, Wang Y. PAD4 is essential for antibacterial innate immunity mediated by neutrophil extracellular traps. J Exp Med (2010) 207:1853-62. doi:10.1084/jem.20100239

24. Wang Y, Li M, Stadler S, Correll S, Li P, Wang D, et al. Histone hypercitrullination mediates chromatin decondensation and neutrophil extracellular trap formation. J Cell Biol (2009) 184:205-13. doi:10.1083/jcb.200806072

25. Nakazawa D, Tomaru U, Yamamoto C, Jodo S, Ishizu A. Abundant neutrophil extracellular traps in thrombus of patient with microscopic polyangiitis. Front Immunol (2012) 3:333. doi:10.3389/fimmu.2012.00333

26. Imamoto $\mathrm{T}$, Nakazawa $\mathrm{D}$, Shida $\mathrm{H}$, Suzuki A, Otsuka N, Tomaru U, et al. Possible linkage between microscopic polyangiitis and thrombosis via neutrophil extracellular traps. Clin Exp Rheumatol (2014) 32:149-50.

27. Knight JS, Zhao W, Luo W, Subramanian V, O'dell AA, Yalavarthi S, et al. Peptidylarginine deiminase inhibition is immunomodulatory and vasculoprotective in murine lupus. J Clin Invest (2013) 123:2981-93. doi:10.1172/JCI67390

28. Bianchi M, Hakkim A, Brinkmann V, Siler U, Seger RA, Zychlinsky A, et al. Restoration of NET formation by gene therapy in CGD controls aspergillosis. Blood (2009) 114:2619-22. doi:10.1182/blood-2009-05-221606

29. Papayannopoulos V, Zychlinsky A. NETs: a new strategy for using old weapons. Trends Immunol (2009) 30:513-21. doi:10.1016/j.it.2009.07.011

30. Steinberg BE, Grinstein S. Unconventional roles of the NADPH oxidase: signaling, ion homeostasis, and cell death. Sci STKE (2007) 2007:e11 doi:10.1126/stke.3792007pe11

31. Clark SR, Ma AC, Tavener SA, McDonald B, Goodarzi Z, Kelly MM, et al. Platelet TLR4 activates neutrophil extracellular traps to ensnare bacteria in septic blood. Nat Med (2007) 13:463-9. doi:10.1038/nm1565

32. Doring Y, Weber C, Soehnlein O. Footprints of neutrophil extracellular traps as predictors of cardiovascular risk. Arterioscler Thromb Vasc Biol (2013) 33:1735-6. doi:10.1161/ATVBAHA.113.301889

33. Grayson PC, Kaplan MJ. At the bench: neutrophil extracellular traps (NETs) highlight novel aspects of innate immune system involvement in autoimmune diseases. JLeukoc Biol (2016) 99:253-64. doi:10.1189/jlb. 5BT0615-247R

34. Lee E, Hirouchi M, Hosokawa M, Sayo H, Kohno M, Kariya K. Inactivation of peroxidases of rat bone marrow by repeated administration of propylthiouracil is accompanied by a change in the heme structure. Biochem Pharmacol (1988) 37:2151-3. doi:10.1016/0006-2952(88)90574-6

35. Huang XR, Holdsworth SR, Tipping PG. Th2 responses induce humorally mediated injury in experimental anti-glomerular basement membrane glomerulonephritis. J Am Soc Nephrol (1997) 8:1101-8.

36. Lang J, Bellgrau D. A T-cell functional phenotype common among autoimmune-prone rodent strains. Scand JImmunol (2002) 55:546-59. doi:10.1046/j.1365-3083.2002.01086.x

37. Neeli I, Khan SN, Radic M. Histone deimination as a response to inflammatory stimuli in neutrophils. J Immunol (2008) 180:1895-902. doi:10.4049/ jimmunol.180.3.1895

38. Luo Y, Arita K, Bhatia M, Knuckley B, Lee YH, Stallcup MR, et al. Inhibitors and inactivators of protein arginine deiminase 4: functional and structural characterization. Biochemistry (2006) 45:11727-36. doi:10.1021/ bi061180d

39. Moscarello MA, Lei H, Mastronardi FG, Winer S, Tsui H, Li Z, et al. Inhibition of peptidyl-arginine deiminases reverses protein-hypercitrullination and disease in mouse models of multiple sclerosis. Dis Model Mech (2013) 6:467-78. doi:10.1242/dmm.010520

40. Willis VC, Gizinski AM, Banda NK, Causey CP, Knuckley B, Cordova KN, et al. N-alpha-benzoyl-N5-(2-chloro-1-iminoethyl)-L-ornithine amide, a protein arginine deiminase inhibitor, reduces the severity of murine collagen-induced arthritis. J Immunol (2011) 186:4396-404. doi:10.4049/ jimmunol.1001620

41. Chumanevich AA, Causey CP, Knuckley BA, Jones JE, Poudyal D, Chumanevich AP, et al. Suppression of colitis in mice by $\mathrm{Cl}$-amidine: a novel peptidylarginine deiminase inhibitor. Am J Physiol Gastrointest Liver Physio (2011) 300:G929-38. doi:10.1152/ajpgi.00435.2010

Conflict of Interest Statement: The authors declare that the research was conducted in the absence of any commercial or financial relationships that could be construed as a potential conflict of interest.

Copyright (c) 2016 Kusunoki, Nakazawa, Shida, Hattanda, Miyoshi, Masuda, Nishio, Tomaru, Atsumi and Ishizu. This is an open-access article distributed under the terms of the Creative Commons Attribution License (CC BY). The use, distribution or reproduction in other forums is permitted, provided the original author(s) or licensor are credited and that the original publication in this journal is cited, in accordance with accepted academic practice. No use, distribution or reproduction is permitted which does not comply with these terms. 